\title{
Quarantots, quarankids, and quaranteens: how research can contribute to mitigating the deleterious impacts of the COVID-19 pandemic on health behaviours and social inequalities while achieving sustainable change
}

\section{Les tout-petits, enfants et ados de la quarantaine : contributions de la recherche à des changements durables pour mitiger les impacts délétères de la pandémie de COVID-19 sur les habitudes de vie et les inégalités sociales}

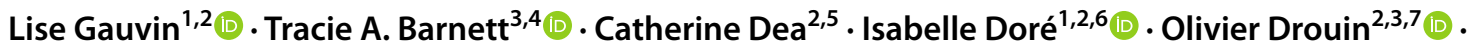 \\ Katherine L. Frohlich ${ }^{2,8}$ (1) Mélanie Henderson ${ }^{2,3,7}$ (1) $\cdot$ Marie-Pierre Sylvestre ${ }^{1,2}(\mathbb{C})$
}

Received: 23 March 2021 / Accepted: 11 August 2021 / Published online: 28 January 2022

(c) The Author(s) under exclusive license to The Canadian Public Health Association 2021

\begin{abstract}
The adoption and maintenance of healthy behaviours including age-appropriate amounts of physical activity, limited sedentary and screen time, and healthy eating are the foundations for youth development and thriving. In reviewing extant evidence, we observe that the COVID-19 pandemic has been associated with marked reductions in physical activity, increased sedentary and screen time, and increased food intake and unhealthy snacking. Deleterious effects in movement behaviours appear to be more pronounced among vulnerable groups and food insecurity has become more widespread. To contribute to mitigating these impacts, we advocate for strengthened evidence-based public health. Towards this end, ongoing surveillance should be intensified and augmented with additional indicators of social inequalities. More importantly, substantial efforts must be devoted to developing, implementing, and evaluating complex interventions aimed at equitably promoting recommended 24-hour movement behaviours and healthy eating guidelines in home, childcare, school, and neighbourhood settings.
\end{abstract}

\section{Résumé}

L'adoption et le maintien de saines habitudes de vie telles des niveaux d'activité physique appropriés pour l'âge, des durées limitées d'activités sédentaires et de temps d'écran, de même qu'une alimentation saine, sont des piliers pour le développement et l'épanouissement des enfants et des jeunes. Après avoir recensé les écrits disponibles, nous constatons que la pandémie de COVID-19 a été associée avec une réduction marquée de l'activité physique, une augmentation de la durée des activités sédentaires et du temps d'écran et une augmentation de la consommation de nourriture et de collations sucrées. Les effets délétères sur les comportements d'activité physique semblent plus prononcés parmi les groupes vulnérables et l'insécurité alimentaire est davantage répandue. Afin de contribuer à atténuer ces impacts, nous proposons le renforcement d'une santé publique fondée sur les données probantes. Nous recommandons une intensification et une bonification des activités de surveillance par l'ajout de nouveaux indicateurs pour mesurer les inégalités sociales de santé. De plus, des efforts considérables doivent être investis dans le développement, l'implantation et l'évaluation d'interventions complexes visant à promouvoir équitablement les directives canadiennes relatives au mouvement sur 24 heures et les saines habitudes alimentaires dans les familles, milieux de garde, écoles et quartiers résidentiels.

Extended author information available on the last page of the article 
Keywords Movement $\cdot$ Diet $\cdot$ Health equity $\cdot$ Child $\cdot$ Adolescent health $\cdot$ COVID-19

Mots-clés Mouvement $\cdot$ alimentation · équité en santé $\cdot$ enfance $\cdot$ adolescence $\cdot$ COVID-19

\section{Introduction}

Promoting healthy eating and optimal 24-hour movement behaviours (i.e., age-appropriate and recommended levels of physical activity (PA), sedentary behaviours (SB) including screen time and sleep) among children 1-17 years are public health imperatives. Acquiring these behaviours during the early years is associated with lower risk of obesity and diabetes and better educational and mental health outcomes (Alamian \& Paradis, 2009; Birch \& Fisher, 1998; Pate et al., 1996; Pronk et al., 2004). Favourable built, social, and service environments (e.g., parks, housing, support from peers and family, neighbourhood trust/social capital, childcare, schools, recreational activities) are the foundations for behavioural and developmental flourishing in children (Goldfeld et al., 2015; Minh et al., 2016; Villanueva et al., 2016). The COVID-19 pandemic led to confinement, physical distancing, and social isolation due to school/childcare closures and community sport and leisure bans. This is thought to have inhibited engagement in healthy behaviours (Alexander \& Shareck, 2021) and likely resulted in socially inequitable impacts of the pandemic on youth (Melbourne Medical School, 2020; Raman et al., 2020). School and childcare settings in normal times offer opportunities for PA (e.g., curricular and extra-curricular activities, outdoor playing, walking/biking to school) and increased food security (e.g., canteen and free healthy meals/snacks for children from disadvantaged households). School and childcare closings have greatly diminished these opportunities, possibly disproportionately impacting vulnerable children. As a result, transient and persistent consequences of the pandemic loom large (Marmot, 2021; Jaeger \& Blaabæk, 2020; LopezBueno et al., 2021; The Lancet Child \& Adolescent Health, 2020; Raman et al., 2020; Rajmil et al., 2021).

We outline the known consequences of the pandemic on health behaviours among children and youth and advocate for strengthened evidence-based public health to contribute to mitigating and possibly reversing the negative impacts of the pandemic.

\section{Movement behaviours}

Scant but compelling research suggests that the pandemic has had numerous deleterious, and unevenly distributed, impacts on health on 24-h movement behaviours (https:// csepguidelines.ca/). Survey data on a large representative sample of Canadian children (5-17 years) revealed lower
PA levels, less time spent outdoors, greater SB (including school and leisure screen time) but more sleep during the pandemic (Moore et al., 2020). Less than 5\% of children and youth met all movement guidelines (Mitra et al., 2020), down from 12.7\% in 2017 (Rhodes et al., 2019). Children were more likely to meet 24 -h movement behaviour guidelines if their parents felt capable of reducing their children's screen time, had higher family income, and were parenting a younger child who was a boy (Guerrero et al., 2020). Neighbourhood factors and age were also important with children (5-11 years vs. adolescents), those living in a house rather than apartment, those living further away from a major road, those residing in high density areas but close to a park, and those from higher income households being more likely to have increased, rather than decreased, outdoor activity (Mitra et al., 2020).

The decrease in PA and increase in SB are observed elsewhere, such as in France, where effects were even more pronounced for children living in urban areas with limited outdoor access (Chambonnière et al., 2021). American children 5-13 years of age engaged in an average of $90 \mathrm{~min}$ of daily school-related sitting time and a staggering $8 \mathrm{~h}$ of leisure-related daily sitting (Dunton et al., 2020). Any PA was more likely to be performed indoors or on neighbourhood streets, and mostly by older children (8-12 years). In Spain, children decreased their level of PA by approximately 90 min per day and increased their screen time by just over $2.5 \mathrm{~h}$ during the pandemic (Medrano et al., 2021). Among children living in Germany, greater duration of SB time prior to the pandemic was predictive of lower PA during the pandemic (Wunsch et al., 2021). Overall, the evidence shows that movement behaviours, including PA and SB, have deteriorated both internationally and at a population level during the pandemic.

\section{Eating habits}

Emerging evidence suggests that the pandemic has had a variety of negative impacts on eating habits, particularly among economically vulnerable groups. A review of 15 studies from the United States, China, Western Europe, and South America showed increased overall food intake and consumption of unhealthy food (Stravilou et al., 2021) and more importantly, a substantial increase in food insecurity - for which there are widely demonstrated adverse consequences (Ke \& Ford-Jones, 2015). In Canada, 14.6\% of Canadian households experienced food insecurity during 
the pandemic (up from 10.5\% in 2017-2018) and more so among households with children (Statistics Canada, 2020). Snacking appears to have increased among Canadian families, but in some cases, healthier behaviours, such as home cooking, also increased (Carroll et al., 2020). A majority of US parents reported doing more home cooking during the pandemic and only a third reported take-out/ delivery food consumption, but an alarming two thirds reported food insecurity (Ferrante et al., 2021). Increases in food insecurity were associated with greater parental concerns about obesity and about one third of families increased their consumption of high-calorie snack foods, sweets/desserts, and fruits in the home (Adams et al., 2020). Parents reported imposing greater restrictions on children's access to certain foods, pressuring children to eat more food, and doing more supervision of children's eating; the practice of pressuring to eat more increased more among food insecure parents (Adams et al., 2020). Unfavourable changes in eating habits such as increased sugary snacking were more likely when parents reported being stressed (Jansen et al., 2021) . In an Italian study, researchers showed that among parents of children under 18 years of age, there was a substantial increase in food insecurity and that children and youth appeared to eat more overall and specifically more junk food (Dondi et al., 2020). Still in Italy, another study showed that among obese children enrolled in a food education program, even though most children ate breakfast regularly and consumed fruit as an afternoon snack, maintenance of healthy eating habits had become more challenging during the pandemic because of increased sedentary behaviour and more frequent reports of hunger (Nicodermo et al., 2021). Finally, a study of 498 families in France showed that more than half of the sample of parents reported a change in at least one dimension of their child's eating habits (Philippe et al., 2021). When changes were reported, they related to increased child appetite, food enjoyment, food responsiveness, and emotional overeating. Parents indicated being more likely to purchase treats and to cook at home. Overall, substantial changes have occurred in children's eating habits, many of which are deleterious. An increase in food insecurity appears to have accompanied the pandemic, but food provision and preparation practices may have been modified differentially with materially advantaged and food secure families doing more home cooking, suggesting a possible increase in the inequity gap.

\section{Where (but especially how) from here}

There is no doubt that ongoing surveillance of PA, ability to play, SB including screen time, and healthy eating should be maintained and even accelerated to examine whether or not some of the apparent impacts of the COVID-19 pandemic are transient or persist over time. There was and remains a glaring gap of evidence for toddlers and preschoolers that must be filled urgently. We believe that extra effort should be made to systematically report the prevalence of healthy eating and movement behaviours as a function of family, school, childcare, neighbourhood, and communitylevel indicators of socio-economic disadvantage and to link these datasets with other data about characteristics of places (Ortigoza et al., 2021). Resulting linked data infrastructures must include classic indicators of socio-economic inequality such as education, income, immigration, and minority status. We suggest adding new indicators of socioeconomic inequality of relevance to the pandemic such as parental loss of employment, additional parental work hours because of employment in frontline occupations, parents having to work from home while caring for children, family members having been infected by the coronavirus, death of a family member due to COVID-19, and dwelling characteristics (i.e., accessibility to outdoor and green spaces, safe parks, household overcrowding, household food insecurity status). At the school level, occurrence of COVID-19 outbreaks, the duration of school closures, implementation of guidance on use of screens, and childcare/school meals and food programs are relevant issues to monitor. At the community level, the duration of the ban on leisure and sport activities, the implementation of park usage restrictions, and changes in the food system are also relevant exposures. Finally, given the widespread adoption of virtual modes of communication, education, work, and entertainment during the pandemic, coupled with the associated risk of increased screen time, we believe that data collection should systematically involve measures designed to tap into digital literacy of youths and their families as well as the digital divide (i.e., access and ability to use digital devices). Existing data infrastructures such as the Canadian Health Survey on Children and Youth (https://www.statcan.gc.ca/eng/survey/household/5233) and the National Longitudinal Study of Children and Youth (https://www23.statcan.gc.ca/imdb/p2SV.pl?Funct ion=getSurvey $\& I d=4631$ ) could plan for additional data collection and data linkages. Entirely new datasets with novel measurement approaches could be added to ongoing pan Canadian data collection to better ascertain healthy eating and movement behaviours among youths aged 0 through 5 years. Collecting clinical data from healthcare providers could also represent an opportunity for improving surveillance of healthy behaviours in the early years.

More importantly, we believe that a vast expansion of population health intervention research (Hawe \& Potvin, 2009) is warranted to better ascertain the reach, acceptability, impact, sustainability, and equity of programs and policies aimed at promoting healthy eating and 24-h movement behaviours and 
at creating/retrofitting home, childcare, school, and neighbourhood environments to render them favourable to these behaviours. Successful interventions are likely to be multicomponent approaches targeting different environmental, organizational, family, and individual determinants of behaviours and, for high-risk children, involve intensive behavioural interventions (e.g., Chaudhary et al., 2020; Dobbins et al., 2013; Gråstén, 2017; Lawson, 2018). Given ongoing and possibly widening social inequalities resulting from the pandemic and the dearth of successful interventions to reduce inequalities, interventions should be adapted and evaluated according to emerging approaches (see Evans et al., 2021; Skivington et al., 2018) . Towards this end, we believe that more participatory research engaging youth and families should be a priority.

\section{Conclusion}

The adoption and maintenance of healthy behaviours are the foundation for youth development and have been shown to track into adulthood. Evidence pointing to detrimental—and unequally detrimental —effects of the pandemic collectively impel us to devote efforts and resources to mitigating declines in healthy behaviours, especially among the most disadvantaged. Given that health behaviours are difficult to change sustainably, we believe that implementation and expansion of evidence-based interventions are required, as are rigorous evaluations of intervention outcomes and widespread dissemination to policy-makers/practitioners. We are poised to invest in a strengthened evidence-based public health and look forward to partnering with other likeminded researchers, practitioners, and citizens.

\section{Les tout-petits, enfants et ados de la quarantaine : contributions de la recherche à des changements durables pour mitiger les impacts délétères de la pandémie de COVID-19 sur les habitudes de vie et les inégalités sociales}

\section{Introduction}

La promotion d'une alimentation saine et d'un comportement de mouvement sain au quotidien chez les enfants et les jeunes de 1 à 17 ans (soit, activité physique, comportement sédentaire, temps d'écran, et sommeil selon les recommandations et la durée appropriées pour l'âge) est un impératif de santé publique. L'acquisition de ces comportements dès le jeune âge est associée à une diminution du risque d'obésité et de diabète et à de meilleurs résultats sur le plan de l'éducation et de la santé mentale (Alamian \& Paradis, 2009; Birch \& Fisher, 1998; Pate et al., 1996; Pronk et al., 2004). Un environnement favorable sur les plans social et organisationnel de même que du bâti (parcs, logements, soutien familial et amical, vie de quartier/vie communautaire, capital social, garderies, écoles, activités de récréation) constitue le pilier du développement et de l'épanouissement des enfants et des jeunes (Goldfeld et al., 2015; Minh et al., 2016; Villanueva et al., 2016). Or, la réponse à la pandémie de la COVID-19 (confinement, distanciation sociale) a entrainé un isolement en raison des fermetures d'écoles/garderies et des interdictions sur les sports et les loisirs communautaires. La pandémie a ainsi eu l'effet de limiter l'engagement dans de saines habitudes de vie (Alexander \& Shareck, 2021) et a vraisemblablement mené à des impacts sociaux inéquitables pour les jeunes (Melbourne Medical School, 2020; Raman et al., 2020). Rappelons qu'en temps normal, l'école et la garderie favorisent l'activité physique (activités scolaires et parascolaires, jeux en plein air, marche/ vélo pour s'y rendre) et offrent une certaine sécurité alimentaire (cantine et repas sains/collations gratuites pour les enfants de milieux défavorisés). Or, les fermetures d'écoles/garderies ont considérablement restreint ces possibilités, affectant peut-être les enfants vulnérables de façon disproportionnée. Il en advient que les conséquences transitoires et persistantes de la pandémie pèsent lourd (Marmot, 2021; Jaeger \& Blaabæk, 2020; LopezBueno et al., 2021; The Lancet Child \& Adolescent Health, 2020; Raman et al., 2020; Rajmil et al., 2021).

Nous décrivons ci-dessous les conséquences connues de la pandémie sur les saines habitudes de vie des enfants et des jeunes, et plaidons en faveur du renforcement d'une santé publique fondée sur des données probantes qui contribuerait à atténuer et à possiblement renverser les effets négatifs de la pandémie.

\section{Mouvement et activité physique}

Malgré le peu de recherche sur le sujet, certaines études suggèrent de façon incontestable des effets délétères, et inégalement répartis suite à la réponse à la pandémie, sur la santé et sur le comportement d'activité physique sur 24 heures (https://csepguidelines.ca/fr/). Une enquête sur un échantillon important et représentatif d'enfants de 5 à 17 ans au Canada au cours de la pandémie signale une diminution de l'activité physique et du temps passé à l'extérieur, une augmentation de l'activité sédentaire (y compris le temps d'écran pour les activités scolaires et loisirs) et une augmentation du sommeil (Moore et al., 2020). Moins de $5 \%$ des enfants et des jeunes 
respectaient toutes les directives en matière de mouvement en 2020 (Mitra et al., 2020) versus 12,7 \% en 2017 (Rhodes et al., 2019). Les enfants étaient plus susceptibles de respecter ces directives sur 24 heures si les parents se sentaient capables de réduire le temps d'écran de l'enfant, s'ils avaient un revenu familial plus élevé, et si l'enfant était un garçon de moins de 12 ans (Guerrero et al., 2020). Le quartier, le genre de logis (maison vs. appartement), et l'âge (5-11 ans vs. adolescent) importent aussi: ceux qui habitaient une maison, loin d'une route principale, dans une région à haute densité mais à proximité d'un parc, et qui provenaient de famille relativement aisée avaient augmenté, plutôt que diminué, l'activité en plein air (Mitra et al., 2020).

La réduction de l'activité physique et l'augmentation du comportement sédentaire liées à la pandémie sont également observées à l'international. En France, l'effet est particulièrement prononcé pour les enfants des zones urbaines aux espaces extérieurs limités (Chambonnière et al., 2021). Aux États-Unis, les enfants d'âge 5 à 13 ans restent assis en moyenne 90 min par jour pour des activités scolaires et un gros huit heures par jour pour se distraire (Dunton et al., 2020). Les activités physiques, quelles qu'elles soient, sont surtout pratiquées par les enfants plus âgés (8-12 ans), généralement soit à l'intérieur soit dans la rue pas loin de la maison. En Espagne, la pandémie témoigne d'une diminution de $90 \mathrm{~min}$ par jour dans l'activité physique et d'une augmentation du temps d'écran d'un peu plus de 2,5 heures par jour (Medrano et al., 2021). En Allemagne, plus les enfants passaient de temps aux activités sédentaires avant la pandémie, moins ils passent de temps aux activités physiques durant la pandémie (Wunsch et al., 2021). Ainsi, dans l'ensemble, les données signalent une détérioration dans les comportements de mouvement (activité physique et sédentaire), à la fois au niveau international et au niveau de la population, depuis le début de la pandémie.

\section{Alimentation}

Les données émergeantes suggèrent un impact négatif de la pandémie sur le régime alimentaire, particulièrement parmi les populations vulnérables sur le plan économique. Un recensement de 15 études en provenance des É-U, de la Chine, de l'Europe occidentale, et de l'Amérique du Sud souligne une augmentation générale de l'apport alimentaire et de la consommation de friandises et de malbouffe (Stravilou et al., 2021). Ajouté à cela, et plus important, il y aurait une augmentation significative de l'insécurité alimentaire entrainant des conséquences adverses largement documentées (Ke \& Ford-Jones, 2015).

Au Canada, 14,6\% des ménages connaissent l'insécurité alimentaire depuis la pandémie (versus 10,5\% en 2017-2018), surtout parmi les familles avec enfants (Statistics Canada, 2020). L'apport en collations semble avoir augmenté; par contre, certaines habitudes alimentaires plus saines, telle la cuisine maison, ont pris le dessus chez certains (Carroll et al., 2020). Aux É-U, la plupart des parents déclarent cuisiner plus souvent pendant la pandémie et seuls un tiers disent consommer des plats à emporter/livraison (Ferrante et al., 2021). Par contre, et voici le facteur inquiétant, deux tiers signalent une insécurité alimentaire. L'augmentation de l'insécurité alimentaire a des retombées sur l'obésité, environ un tiers des familles ayant augmenté leur consommation en collations riches en calories, en sucreries/desserts, et en fruits (Adams et al., 2020). Malgré que les parents imposent aux enfants des restrictions à l'accès de certains aliments et surveillent davantage leur alimentation, ils insistent parfois à ce que l'enfant mange plus; cette insistance est particulièrement accrue chez les parents en situation d'insécurité alimentaire (Adams et al., 2020). D'ailleurs, il a été démontré que les habitudes alimentaires s'empirent souvent quand les parents se disent stressés; ex. l'apport important en sucreries (Jansen et al., 2021).

En Italie, les chercheurs démontrent une recrudescence de l'insécurité alimentaire chez les parents d'enfants de moins de 18 ans; de plus, l'apport alimentaire global et plus particulièrement celui de la malbouffe, se trouvent augmentés chez les enfants (Dondi et al., 2020). Toujours en Italie, une autre étude, celle-ci parmi des enfants obèses inscrits dans un programme d'éducation alimentaire, souligne que même si la plupart consommaient régulièrement un petit-déjeuner et ensuite un fruit en après-midi, le maintien des habitudes saines devenait de plus en plus difficile pendant la pandémie en raison de l'augmentation du train de vie sédentaire et de la fréquence d'une sensation de faim (Nicodermo et al., 2021). Enfin, en France, plus de la moitié des 498 familles recensées rapportent un changement concernant au moins une facette de l'alimentation de leurs enfants, soit l'appétit, la dégustation, la sensibilité à la nourriture, ou la suralimentation émotionnelle (Philippe et al., 2021). Les parents étaient plus aptes à acheter des friandises et à cuisiner à la maison.

Pour résumer, depuis la pandémie, de nombreux changements ont affecté les habitudes alimentaires des enfants, et ce, souvent avec un impact potentiellement négatif. L'insécurité alimentaire semble avoir accompagné la pandémie. De plus, l'approvisionnement alimentaire et la préparation de la nourriture semblent se différencier sur le plan socioéconomique, les familles plus avantagées cuisinant des petits plats maison. Ainsi, il y a suggestion d'un écart grandissant des inégalités sociales.

\section{Et maintenant, où et particulièrement comment?}

Il n'y a aucun doute qu'il est important de maintenir, et peut-être même d'accélérer, les enquêtes sur l'activité physique, le jeu des enfants, le comportement sédentaire y compris le temps d'écran, et l'alimentation saine, afin de déterminer 
si les impacts observés de la réponse à la pandémie de la COVID-19 sont transitoires ou persistent à travers le temps. De plus, il y avait et continue à y avoir un manque flagrant de données probantes sur les tout-petits et les enfants d'âge préscolaire, lacune qui doit être adressée de toute urgence. Nous sommes convaincus qu'un effort complémentaire doit être entrepris pour signaler systématiquement la prévalence des comportements alimentaires et des comportements en matière de mouvement, en fonction des caractéristiques de la famille, de l'école, de la garderie, du lieu de résidence, et des indicateurs communautaires du statut socio-économique, et de jumeler ces bases de données avec d'autres données sur les caractéristiques locales/régionales (Ortigoza et al., 2021). Les banques de données ainsi construites devront inclure les indicateurs classiques de l'inégalité socio-économique, tels que l'éducation, le revenu, et le statut d'immigration et de minorité ethnique. Nous suggérons l'ajout de nouveaux indicateurs d'inégalité socio-économique en lien avec la pandémie, tels que la perte d'emploi, les heures de travail supplémentaire dus à un emploi de première ligne, le télétravail à domicile avec des enfants à la maison, la COVID-19 chez un membre de la famille, la décès d'un membre de la famille en raison de la COVID-19, les caractéristiques du logement (accessibilité à l'extérieur et aux espaces verts, parcs sécuritaires, logement à l'étroit (famille nombreuse)), et le statut d'insécurité alimentaire familiale. Sur le plan scolaire, il serait pertinent de surveiller les éclosions de COVID-19, les fermetures d'écoles et leur durée, l'implantation des directives sur le temps d'écran, et les programmes d'alimentation pour les repas/collations à l'école/ garderie. Sur le plan communautaire, il faudrait examiner les interdictions des activités ludiques/loisirs/sportives et leur durée, l'implantation des restrictions sur les parcs, et les changements affectant l'approvisionnement alimentaire. Et finalement, avec la mise en place et l'acceptation générale des modes virtuels de communication (enseignement, télétravail, distraction/spectacles durant la pandémie), et du temps d'écran qui y est forcément attaché, il nous parait évident que la collecte de données doit inclure systématiquement des mesures sur le virage numérique/connaissances numériques des jeunes et des familles; ainsi que des mesures de fracture numérique, soit l'accès et la capacité d'utilisation des modes numériques. Les infrastructures existantes telles que l'Enquête canadienne sur la santé des enfants et des jeunes (https://www.statcan. gc.ca/fr/enquete/menages/5233)et l'Enquête longitudinale nationale sur les enfants et les jeunes (https://www23.statc an.gc.ca/imdb/p2SV_f.pl?Function=getSurvey\&Id=4631) pourraient élargir leurs banques de données pour accommoder des collectes et des jumelages complémentaires. Sur le plan des enfants de bas âge ( $0-5$ ans), de nouvelles bases de données comprenant des mesures innovantes pourraient être ajoutées à la collecte de données pancanadiennes sur l'alimentation saine et les comportements de mouvement et d'activité physique. Également, la collecte des données cliniques auprès des intervenants/professionnels de la santé saurait améliorer le suivi des comportements sains.

Ce qui est encore plus important, nous préconisons qu'une vaste expansion de la recherche interventionnelle en santé des populations (Hawe \& Potvin, 2009) soit justifiée pour mieux déterminer la portée, l'acceptabilité, l'impact, la durabilité et l'équité des programmes et des politiques visant à promouvoir une alimentation saine et un comportement de mouvement sain au quotidien. Ceci inclut l'innovation et/ou l'adaptation d'un milieu propice à ces comportements à la maison, à la garderie, à l'école, et dans les lieux communautaires. Vraisemblablement, les interventions optimales comprendront une approche multifacette à plusieurs volets, ciblant différents déterminants environnementaux, organisationnels, familiaux, et individuels du comportement et, pour les enfants à risque, comprenant des interventions comportementales intensives (ex. Chaudhary et al., 2020; Dobbins et al., 2013; Gråstén, 2017; Lawson, 2018). Compte tenu des inégalités sociales persistantes et possiblement grandissantes en raison de la pandémie, et du manque d'interventions efficaces pour réduire les inégalités, ces interventions devront être adaptées et évaluées en fonction d'approches émergentes (voir Evans et al., 2021; Skivington et al., 2018). Ce qui confirme pour nous qu'une recherche plus participative impliquant les jeunes et les familles devrait être considérée prioritaire.

\section{Conclusion}

L'adoption et le maintien des comportements sains sont à la base du développement des jeunes, et leurs effets retentissent de façon démontrable à l'âge adulte. À cela, certaines évidences indiquent des effets adverses —et inégalement adverses—de la pandémie, nous incitant collectivement à consacrer efforts et ressources pour atténuer le déclin des comportements sains, en particulier chez les individus et les milieux les plus défavorisés. Étant donné la difficulté de changer le comportement de façon durable, nous favorisons l'implantation et l'expansion d'interventions fondées sur des données probantes, ainsi que l'évaluation rigoureuse des résultats de ces interventions et la diffusion à grande échelle aux décideurs/praticiens. Nous sommes prêts à investir dans le renforcement d'une santé publique fondée sur des données probantes, et c'est avec plaisir que nous cherchons à collaborer avec d'autres chercheurs, praticiens, et citoyens qui partagent la même vision.

Acknowledgements TAB holds a Chercheur-boursier Senior award from FRQS; ID holds a Chercheur-boursier J1 award from FRQS; OD holds a Chercheur-boursier-clinicien J1 salary award from the Fonds de recherche Québec - santé (FRQS); KLF is a joint holder of the Myriagone 
UdM-McConnell Chair on Youth Knowledge Mobilisation; MH holds a Chercheur-boursier-clinicien J2 award from FRQS and is recipient of the 2019 Canadian Society of Endocrinology and Metabolism's Young Investigator Award; MPS holds a Chercheur-boursier J2 award from FRQS. French translation: Danielle Buch, Medical writer, Research.

Author contributions LG conceptualized and wrote the first draft. TAB, $\mathrm{CD}$, ID, OD, KLF, MH, and MPS provided critical revisions and intellectual content. LG integrated revisions and content proposed by coauthors.

Availability of data and material Not applicable.

Code availability Not applicable.

\section{Declarations}

Ethics approval Not applicable.

Consent to participate Not applicable.

Consent for publication Not applicable.

Conflict of interest LG is Associate Scientific Director for Population Health and Theme Leader on Innovation \& Evaluation at the Centre de recherche du CHUM and a Member of the Institute Advisory Board of the Institute for Population and Public Health of the Canadian Institutes of Health Research; MPS provides statistical consultation at the Institut de Cardiologie de Montréal.

\section{References/Références}

Adams, E. L., Caccavale, L. J., Smith, D., \& Bean, M. K. (2020). Food insecurity, the home food environment, and parent feeding practices in the era of COVID-19. Obesity, 28, 2056-2063.

Alamian, A., \& Paradis, G. (2009). Clustering of chronic disease behavioral risk factors in Canadian children and adolescents. Preventive Medicine, 48(5), 493-499.

Alexander, S.A., \& Shareck, M. (2021). Widening the gap? Unintended consequences of health promotion measures for young people during COVID-19 lockdown. Health Promotion International 1-13. https://doi.org/10.1093/heapro/daab015

Birch, L. L., \& Fisher, J. O. (1998). Development of eating behaviors among children and adolescents. Pediatrics, 101(3 Pt 2), 539-549.

Carroll, N., Sadowski, A., Laila, A., Hruska, V., Nixon, M., Ma, D. W. L., Haines, J., \& on behalf of the Guelph Family Health Study. (2020). The impact of COVID-19 on health behavior, stress, financial and food security among middle to high income Canadian families with young children. Nutrients, 12, 2352.

Chambonnière, C., Lambert, C., Fearnbache, N., Tardieu, M., Fillon, A., Genin, P., Larra, B., Melsens, P., Bois, J., Pereira, B., Tremblay, A., Thivel, D., \& Duclos, M. (2021). Effect of the COVID-19 lockdown on physical activity and sedentary behaviors in French children and adolescents: New results from the ONAPS national survey. European Journal of Integrative Medicine, 43, 101308.

Chaudhary, A., Sudzina, F., \& Mikkelsen, B. E. (2020). Promoting healthy eating among young people-a review of the evidence of the impact of school-based interventions. Nutrients, 12(9), 2894.

Canadian Health Survey on Children and Youth (CHSCY). https:// www23. statcan.gc.ca/imdb/p2SV.p1?Function=getSurvey\& SDDS=5233, Consulted on September 1, 2021.

Dobbins, M., Husson, H., DeCorby, K., \& LaRocca, R. L. (2013). School-based physical activity programs for promoting physical activity and fitness in children and adolescents aged 6 to 18 . Cochrane Database of Systematic Reviews, Issue 2. https://doi. org/10.1002/14651858.CD007651.pub2.

Dondi, A., Candela, E., Morigi, F., Lenzi, J., Pierantoni, L., \& Lanari, M. (2020). Parents' perception of food insecurity and of its effects on their children in Italy six months after the COVID-19 pandemic outbreak. Nutrients, 13(1), 121.

Dunton, G. F., Do, B., \& Wang, S. D. (2020). Early effects of the COVID-19 pandemic on physical activity and sedentary behavior in children living in the U.S. BMC Public Health, 20, 1351.

Evans, R. E., Moore, G., Movsisyan, A., Rehfuess, E., \& The ADAPT Panel. (2021). How can we adapt complex population health interventions for new contexts? Progressing debates and research priorities. Journal of Epidemiology \& Community Health, 75, 40-45.

Ferrante, M. J., Goldsmith, J., Tauriello, S., Epstein, L. H., Leone, L. A., \& Anzman-Frasca, S. (2021). Food acquisition and daily life for U.S. families with 4- to 8-year-old children during COVID-19: Findings from a nationally representative survey. International Journal for Environmental Research \& Public Health, 18, 1734.

Goldfeld, S., Woolcock, G., Katz, I., Tanton, R., Brinkman, S., O'Connor, E., Mathews, T., \& Giles-Corti, B. (2015). Neighbourhood effects influencing early childhood development: Conceptual model and trial measurement methodologies from the Kids in Communities study. Social Indicators Research, 120, 197-212.

Gråstén, A. (2017). School-based physical activity interventions for children and youth: Keys for success. Journal of Sport and Health Science, 6(3), 290-291.

Guerrero, M. D., Vanderloo, L. M., Rhodes, R. E., Faulkner, G., Moore, S. A., \& Tremblay, M. S. (2020). Canadian children's and youth's adherence to the 24-h movement guidelines during the COVID-19 pandemic: A decision tree analysis. Journal of Sport and Health Science, 9, 313-321.

Hawe, P., \& Potvin, L. (2009). What is population health intervention research? Canadian Journal of Public Health, 100(1), Suppl I8-14.

Jaeger, M. M., \& Blaabæk, E. H. (2020). Inequality in learning opportunities during Covid-19: Evidence from library takeout. Research in Social Stratification and Mobility, 68, 100524.

Jansen, E., Thapaliya, G., Aghababian, A., Sadler, J., Smith, K., \& Carnell, S. (2021). Parental stress, food parenting practices and child snack intake during the COVID-19 pandemic. Appetite, 161, 105119.

Ke, J., \& Ford-Jones, E. L. (2015). Food insecurity and hunger: A review of the effects on children's health and behaviour. Paediatric Child Health, 20(2), 89-91.

Lawson, H. A. (Ed.). (2018). Redesigning physical education: An equity agenda in which every child matters. London: Taylor \& Francis Group.

Lopez-Bueno, R., Lopez-Sanchez, G. F., Casajús, J. A., Calatayud, J., Tully, M. A., \& Smith, L. (2021). Potential health-related behaviors for pre-school and school-aged children during COVID-19 lockdown: A narrative review. Preventive Medicine, 143, 106349.

Marmot, M. (2021). Sir Michael Marmot on COVID-19, inequality and the future of society. The Lancet Voice Podcast. March 5, Season 2, Episode 5. https://www.thelancet.com/the-lancet-voice?dgcid=hubspot_ email_newsletter_tlcoronavirus20\&_hsenc=p2ANqtz-_xcsPIS0as0 szk423T8jzBrXUsYo_VOk6680rGOGpj6lEuUHplhrje4P9zi3tr8F jtRKfDZGILWYxgqGYyFIQFiH5ftw\&_hsmi=88296112.

Medrano, M., Cadenas-Sanchez, C., Oses, M., Arenaza, L., Amasene, M., Labayen, I. (2021). Changes in lifestyle behaviours during the COVID-19 confinement in Spanish children: A longitudinal analysis from the MUGI project. Pediatric Obesity, 16(4), e12731.

Melbourne Medical School. (2020). COVID-19 kids research evidence update: What the Melbourne children's clinicians, scientists, epidemiologists, and medical students have been reading this week. Weekly Update No.30 19th November, https://medicine.unime lb.edu.au/_data/assets/pdf_file/0005/3542873/Covid-19_Kids_ Research_evidence_update_week30_19November_2020.pdf. 
Minh, A., Muhajarine, N., Janus, M., Brownell, M., \& Guhn, M. (2016). A review of neighborhood effects and early child development: How, where, and for whom, do neighborhoods matter? Health and Place, 46, 155-174.

Mitra, R., Moore, S. A., Gillespie, M., Faulkner, G., Vanderloo, L. M., Chulak-Bozzer, T., Rhodes, R. E., Brussoni, M., \& Tremblay, M. S. (2020). Healthy movement behaviours in children and youth during the COVID-19 pandemic: Exploring the role of the neighbourhood environment. Health and Place, 65, 102418.

Moore, S. A., Faulkner, G., Rhodes, R. E., Brussoni, M., ChulakBozzer, T., Ferguson, L. J., Mitra, R., O'Reilly, N., Spence, J. C., Vanderloo, L. M., \& Tremblay, M. S. (2020). Impact of the COVID-19 virus outbreak on movement and play behaviours of Canadian children and youth: A national survey. International Journal of Behavioral Nutrition and Physical Activity, 17, 85.

Nicodermo, M., Spreghini, M. R., Manco, L. M., Wietrzykowska Sforza, R., \& Morino, G. (2021). Childhood obesity and COVID19 lockdown: Remarks on eating habits of patients enrolled in a food-education program. Nutrients, 13, 383.

National Longitudinal Survey of Children and Youth (NLSCY). https:// www23.statcan.gc.ca/imdb/p2SV.pl?Function=getSurvey\&Id=4631.

Ortigoza, A. F., Tapia Granados, J. A., Miranda, J. J., Alazraqui, M., Higuera, D., Villamonte, G., de Lima Friche, A. A., Gutierrez, T. B., \& Diez Roux, A. V. (2021). Characterising variability and predictors of infant mortality in urban settings: Findings from 286 Latin American cities. Journal of Epidemiology \& Community Health, 75, 264-270.

Pate, R. R., Baranowski, T., Dowda, M., \& TROST, S. G. . (1996). Tracking of physical activity in young children. Medicine \& Science in Sports \& Exercise, 28(1), 92-96.

Philippe, K., Chabanet, C., Issanchou, S., \& Monnery-Patris, S. (2021). Child eating behaviors, parental feeding practices and food shopping motivations during the COVID-19 lockdown in France: (How) did they change? Appetite, 161, 105132.

Pronk, N. P., Anderson, L. H., Crain, A. L., Martinson, B. C., O’Connor, P. J., Sherwood, N. E., \& Whitebird, R. R. (2004). Meeting recommendations for multiple healthy lifestyle factors. Prevalence, clustering, and predictors among adolescent, adult, and senior health plan members. American Journal of Preventive Medicine, 27(Suppl 2), 25-33.

Rajmil, L., Hjern, A., Boran, P., \& on behalf of International Society for Social Pediatrics \& Child Health (ISSOP) and International Network for Research on Inequalities in Child Health (INRICH)
COVID-19 Working Group. (2021). Impact of lockdown and school closure on children's health and well-being during the first wave of COVID-19: A narrative review. BMJ Paediatrics Open, 5, e001043.

Raman, S., Harries, M., Nathawad, R., Kyeremateng, R., Seth, R., Lonne, B., \& on behalf of International Society for Social Pediatrics \& Child Health (ISSOP) COVID-19 Working Group. (2020). Where do we go from here? A child rights-based response to COVID-19. BMJ Paediatrics Open, 4, e000714.

Rhodes, R. E., Spence, J. C., Berry, T., et al. (2019). Parental support of the Canadian 24-hour movement guidelines for children and youth: Prevalence and correlates. BMC Public Health, 19(1), 1385.

Skivington, K., Matthews, L., Craig, P., Simpson, S., \& Moore, L. (2018). Developing and evaluating complex interventions: Updating Medical Research Council guidance to take account of new methodological and theoretical approaches. The Lancet, 392, S2. https://doi.org/10.1016/S0140-6736(18)32865-4.

Statistics Canada. (2020). Food insecurity during the COVID-19 pandemic, May 2020. Published: June 24 2020. https://www150.statc an.gc.ca/n1/pub/45-28-0001/2020001/article/00039-eng.htm, consulted on September 12021.

Stravilou, A., Kapsali, E., Panagouli, E., Thirios, A., Polychronis, K., Bacopoulou, F., Psaltopoulou, T., Tsolia, M., Sergentanis, T. N., \& Tsitsika, A. (2021). Obesity in children and adolescents during COVID-19 pandemic. Children, 8, 135.

The Lancet Child \& Adolescent Health. (2020). Growing up in the shadow of COVID-19. The Lancet: Child \& Adolescent Health, 4(12), 853.

Villanueva, K., Badland, H., Kvalsvig, A., O’Connor, M., Christian, H., Woolcock, G., Giles-Corti, B., \& Goldfeld, S. (2016). Can the neighborhood built environment make a difference in children's development? Building the research agenda to create evidence for place-based children's policy. Academic Pediatrics, 16(1), 10-19.

Wunsch, K., Nigg, C., Niessner, C., Schmidt, S. C. E., Oriwol, D., Hanssen-Doose, A., Burchartz, A., Eichsteller, A., Kolb, S., Worth, A., \& Woll, A. (2021). The impact of COVID-19 on the interrelation of physical activity, screen time and health-related quality of life in children and adolescents in Germany: Results of the Motorik-Modul Study. Children, 8, 98.

Publisher's note Springer Nature remains neutral with regard to jurisdictional claims in published maps and institutional affiliations.

\section{Authors and Affiliations}

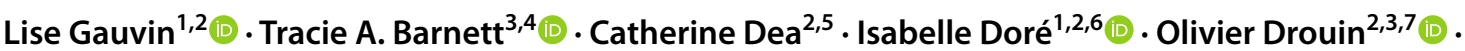 Katherine L. Frohlich ${ }^{2,8}\left(\right.$ D $\cdot$ Mélanie Henderson ${ }^{2,3,7}\left(\right.$ Marie-Pierre Sylvestre $^{1,2}(\mathbb{D}$}

\section{Lise Gauvin}

lise.gauvin.2@umontreal.ca

1 Centre de recherche du Centre Hospitalier de l'Université de Montréal (CRCHUM), 850 Rue Saint-Denis, Montréal, Québec H2X 0A9, Canada

2 Département de médecine sociale et préventive, École de Santé Publique de l'Université de Montréal (ESPUM), P.O. Box 6128, Centre-ville Station, Montréal, Québec H3C 3J7, Canada

3 Centre de recherche du Centre Hospitalier Universitaire Sainte-Justine (CRCHUSJ), Montreal, Québec, Canada

4 Department of Family Medicine, Faculty of Medicine, McGill University, Montreal, Québec, Canada
5 Direction Régionale de Santé Publique, Centre Intégré Universitaire de Santé et Services Sociaux du Centre-Sud-deL'Île-de-Montréal, Montréal, Québec, Canada

6 École de Kinésiologie et des Sciences de l'Activité Physique (EKSAP), Faculté de médecine, Université de Montréal, Montréal, Québec, Canada

7 Département de pédiatrie, Faculté de médecine, Université de Montréal, Montréal, Québec, Canada

8 Centre de Recherche en Santé Publique (CReSP), Montréal, Québec, Canada 